\title{
MARCO CONCEPTUAL PARA EL FORTALECIMIENTO DE LOS SISTEMAS DE INFORMACIÓN EN SALUD EN EL PERÚ
}

\author{
FRAMEWORK FOR THE STRENGTHENING OF HEALTH INFORMATION SYSTEMS IN PERU \\ Walter H. Curioso ${ }^{1, a}$, Elizabeth Espinoza-Portilla ${ }^{2, b}$
}

\author{
Department of Biomedical Informatics and Medical Education, School of Medicine, University of Washington. Seattle, Washington, EE. UU. \\ Instituto de Gobierno y de Gestión Pública. Universidad San Martín de Porres. Lima, Perú. \\ Médico cirujano, magíster en Salud Pública y doctor en Informática Biomédica. \\ Médico cirujano, magíster en Demografía y Población, máster en Estudios Políticos Aplicados. \\ Recibido: 20-05-15; Aprobado: 01-06-15
}

\section{RESUMEN}

En este artículo se presentan los componentes esenciales y políticas más relevantes con respecto al marco conceptual para fortalecer los sistemas de información en salud en el Perú. Se presentan, además, las principales políticas, acciones y estrategias más significativas realizadas en el campo de la salud electrónica en el Perú. Los sistemas de información en salud en el Perú cumplen un rol clave y se espera lograr un sistema de información integrado e interoperable que permita que la información en salud sea lo más completa, eficiente, de calidad y esté disponible oportunamente, para lograr mejorar la calidad de vida de las personas y permitir una modernización significativa de la salud pública en el marco de la reforma en salud en el Perú.

Palabras clave: Sistemas de información en salud; Informática médica; Telesalud; Tecnologías de información (fuente DeCS BIREME).

\begin{abstract}
In this article we present the essential components and policies that are most relevant regarding the conceptual framework to strengthen the health information systems in Peru. The article also presents the main policies, actions and strategies made in the field of electronic health in Peru that are most significant. The health information systems in Peru play a key role and are expected to achieve an integrated and interoperable information system. This will allow health information to be complete, efficient, of good quality and available in a timely manner to achieve better quality of life for people and allow meaningful modernization of public health in the context of health reform in Peru.
\end{abstract}

Key words: Health information systems; Medical informatics; Telehealth; Information technology (source: MeSH NLM).

\section{INTRODUCCIÓN}

Un sistema de salud necesita de una organización eficiente, construida sobre un robusto sistema que permita el acceso oportuno a información de calidad acerca de la salud de la persona y de la población. Para lograr estos objetivos, se necesitan mejorar procesos, integrar y evaluar nuevas y apropiadas tecnologías y desplegar una óptima infraestructura, además de garantizar su mantenimiento y monitoreo ${ }^{(1)}$.

Si bien existen normativas relacionadas al campo de las tecnologías de información y comunicación (TIC) y salud que involucran temas de alcance institucional y/o sectorial (2), muchas de ellas fueron propuestas atendiendo a cierta necesidad, y hasta el momento solo existe publicado un documento técnico que establece el marco conceptual para el fortalecimiento en sistemas de información y tecnologías de información y de comunicación en el Ministerio de Salud, aprobado por Resolución Ministerial 297-2012/MINSA ${ }^{(3)}$.

Dicho marco tiene como objetivo integrar los mecanismos y estándares de información del sector salud y surge como herramienta gráfica para la integración y coordinación de las acciones relacionadas con los sistemas de información y las tecnologías de información y de comunicación ${ }^{(3)}$. El marco conceptual toma como referencias documentos técnicos de la Organización Mundial de la Salud (4) y la International Organization for Standardization ${ }^{(5)}$.

Es por ello que, a fin de conducir el proceso de planeamiento estratégico de los sistemas de información en salud, es necesario identificar los componentes relacionados al fortalecimiento de los sistemas de información en salud, con la misión de contribuir a mejorar la salud de la población a través de una 
gestión oportuna, eficiente y confiable de información estandarizada, que pueda luego ser compartida para una apropiada toma de decisiones en salud.

El objetivo de este artículo es identificar los principales componentes y describir el rol y aplicaciones que desempeñan en el ecosistema de los sistemas de información en salud y de las TIC en salud en el Perú.

\section{PRINCIPALES COMPONENTES DEL MARCO CONCEPTUAL}

Para facilitar la comprensión de los conceptos se ha establecido una analogía entre un sistema de información y una vivienda ${ }^{(6)}$. Los principales elementos se muestran en la Figura 1. El marco conceptual involucra los siguientes cuatro grandes componentes: infraestructura, infoestructura, la telesalud y el proceso de planeamiento, ejecución, monitoreo y evaluación.

\section{INFRAESTRUCTURA: TECNOLOGÍAS DE INFORMACIÓN Y COMUNICACIÓN}

Es la base que da soporte al sistema de salud electrónica y se compone de los siguientes elementos:

\section{Infraestructura tecnológica}

Es el conjunto de elementos de hardware (equipos de cómputo, impresoras, servidores, red de datos, etc.), software (sistemas operativos, bases de datos, lenguajes de programación, herramientas de administración, etc.) y servicios (soporte técnico, seguros, comunicaciones, etc.) que en conjunto dan soporte a las aplicaciones (sistemas informáticos). Adicionalmente, este componente incluye la red de comunicaciones ${ }^{(6)}$. Es preciso señalar que tanto el software, el hardware y la red de comunicaciones confluyen en lo que se conoce como centros de procesamiento de datos que deben disponer de apropiadas medidas de acondicionamiento y medidas de seguridad ${ }^{(6)}$.

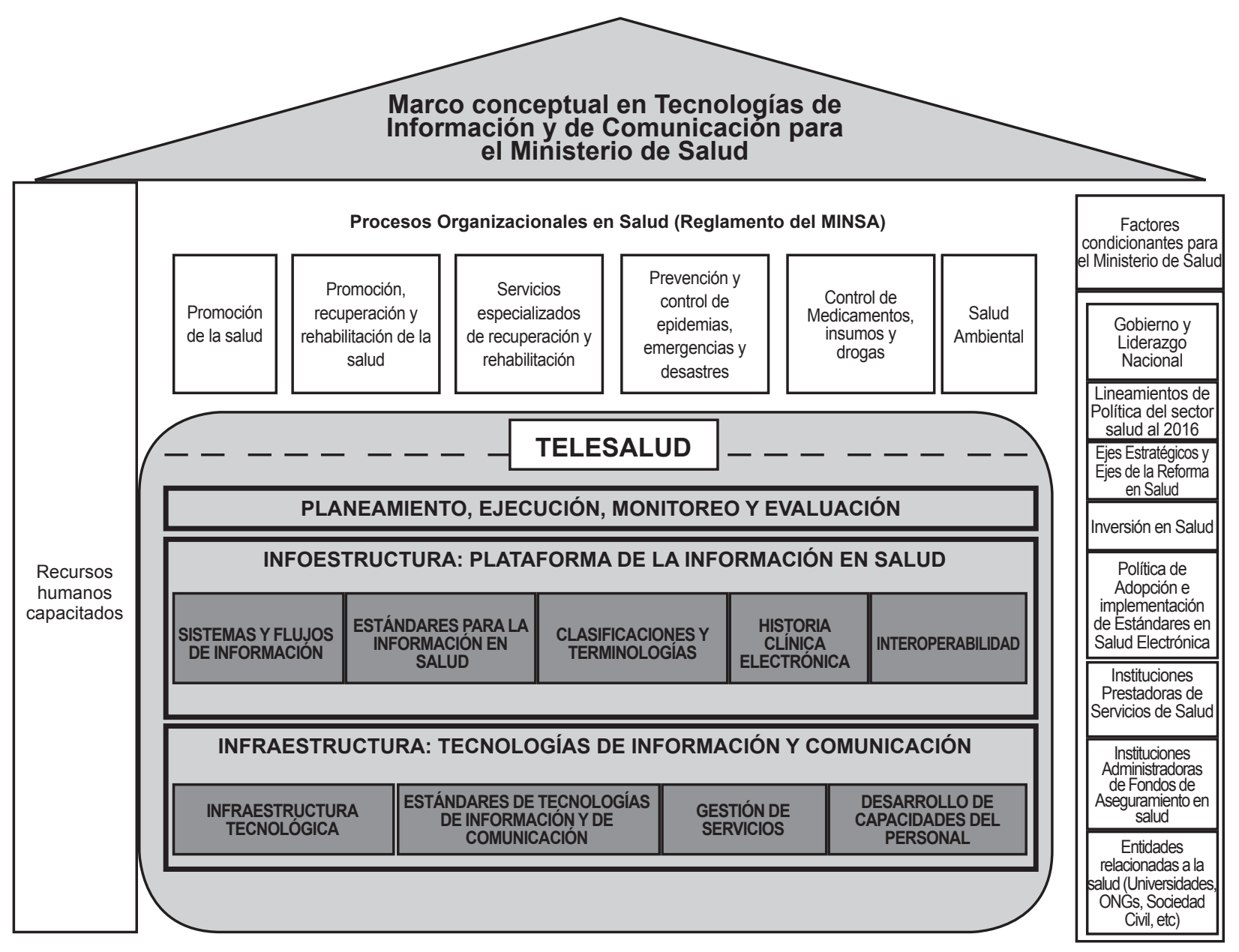

Figura 1. Esquema del marco conceptual en TIC para el Ministerio de Salud de Perú 
Para aprovechar al máximo los beneficios de la tecnología, el Estado requiere una apropiada infraestructurafísica que la soporte a través de una gran red de transporte y acceso que le permita integrarse a otras redes. Con la Ley 29904 "Ley de promoción de la Banda Ancha y construcción de la Red Dorsal Nacional de Fibra Óptica" la cual fue reglamentada con el Decreto Supremo 014-2013-MTC, se busca que el Estado cuente con una red de acceso, la Red Nacional del Estado Peruano (REDNACE), que pueda utilizar eficientemente para el desarrollo de sus actividades. Las áreas de aplicación de proyectos que se potenciarian con fibra óptica son: telesalud, telemedicina, teleeducación, videoconferencias, imágenes satelitales, procesamiento de imágenes, nanotecnología, computación paralela, servicios que permitan compartir recursos, entre otros ${ }^{(7)}$.

\section{Estándares de tecnologías de información y de comunicación}

Es el conjunto de normas, políticas, reglas o procedimientos, a los que debe ajustarse las tecnologías de información y de comunicación. En salud, la necesidad de trabajar con estándares es fundamental.

Según la Resolución Ministerial 381-2008-PCM que aprueba los "Estándares y Especificaciones de Interoperabilidad del Estado Peruano" se promueve la adopción, por un lado, de los estándares abiertos en las especificaciones técnicas de las soluciones informáticas del Estado, y por otro lado se promueven los estándares internacionales. Dicha resolución establece que los estándares y patrones establecidos por el Estado deben mantener la mayor correspondencia posible con los estándares internacionales de reconocimiento mundial, regional y nacional, según esté disponible. Asimismo, se considera en forma prioritaria los estándares de la International Organization for Standardization (ISO), especialmente los que se han constituido en Norma Técnica Peruana por el Instituto Nacional de Defensa de la Competencia y de la Protección de la Propiedad Intelectual (INDECOPI). Además, se especifica que el desarrollo de metadatos del Estado debe estar basado en estándares internacionales.

\section{Gestión de servicios}

Se basa en procesos y se enfoca en alinear los servicios de TIC poniendo énfasis en los beneficios que se espera perciba el cliente final. Involucra la mejora continua de los procesos como las operaciones en TIC, mesa de ayuda, desarrollo de software, gestión del conocimiento en TIC, entre otros.
La eficacia, eficiencia y efectividad en la satisfacción de las necesidades del usuario final dependen de la calidad con la que se desarrollen e implemente un sistema de información y de su correcto uso y mantenimiento ${ }^{(6)}$.

El eje central de la gestión de los servicios es la creación de equipos de trabajo armónicos y estables en los que participen profesionales multidisciplinarios con una asignación clara de los roles y responsabilidades de cada uno que pueden incluir: personal clínico (para el liderazgo del proyecto, definición de requerimientos, pruebas de validación y uso), personal técnico (para el desarrollo de software, implementación de hardware, despliegue y mantenimiento), gestores de proyectos (coordinación general), directivos (para la toma de decisiones) y profesionales externos a la organización ${ }^{(6)}$

\section{Desarrollo de capacidades del personal}

El desarrollo de capacidades es el proceso de internalización de conocimiento y habilidades que permite a las personas resolver problemas, desempeñar funciones y tomar decisiones orientadas a mejorar los resultados en las acciones que realicen. El desarrollo de capacidades del personal de la organización es una pieza fundamental en los proyectos que involucran sistemas de información en salud. Este desarrollo de capacidades debe incluir materias tan diversas como métodos de gestión de proyectos, estándares, e incluso normativas a las que están sujetas las instituciones de salud. Además, es importante planificar procesos de desarrollo de capacidades inicial y luego un programa de capacitación continua. La primera debe ser impartida al inicio de la implementación de un nuevo sistema y también cuando se incorpora un nuevo personal. La segunda se debe emplear tanto para refrescar conocimientos como para capacitar al personal en el manejo apropiado y eficiente de las nuevas funcionalidades ${ }^{(6)}$.

\section{INFOESTRUCTURA: PLATAFORMA DE LA INFORMACIÓN EN SALUD.}

La infoestructura en salud no solo se refiere al uso de la información y las comunicaciones en salud, sino también a la información disponible, ya sea como texto, sonidos, imágenes e incluso datos, así como a todas las aplicaciones y software necesario para acceder, manipular, organizar y sistematizar la información, además de las políticas dadas para el uso apropiado de la información, y también el componente 
humano y organizacional de quienes gestionan la información y usan la infraestructura. Sus objetivos son el desarrollar mecanismos que permitan a los usuarios acceder oportunamente a la información en salud para una toma de decisiones apropiada y de calidad, de facilitar el trabajo de los profesionales de salud a través de las tecnologías, y el de crear un red integrada de historias clínicas electrónicas a través de todos los procesos de salud ${ }^{(8)}$.

Uno de los grandes desafíos incluye el desarrollo de una infoestructura nacional, similar a lo desarrollado por Canadá (9), para apoyar el intercambio de datos en base a identificadores estándares de datos y normas técnicas para lograr una óptima interoperabilidad de los sistemas de información en salud, y que faciliten un eficiente intercambio prestacional.

Los componentes de infoestructura que considerar deben incluir un análisis de procesos y flujos de información, además, se debe considerar los identificadores estándares de datos en salud (IEDS), especificados en el Decreto Supremo 024-2005-SA, que contiene la descripción técnico-normativa de la identificación estándar de datos básicos en salud.

El mapa de procesos debe identificar y agrupar los procesos de la organización de acuerdo a los niveles de la toma de decisiones (estratégico, operativo y de apoyo).

Otro elemento de la infoestructura son las clasificaciones y terminologías. Según el Decreto Supremo 024-2005-SA, para la clasificación estándar del procedimiento médico se adopta la clasificación del Current Procedural Terminology (CPT) de la Asociación Médica Americana; y según la R.M. 553-2002-SA se oficializa el uso de la Clasificación Estadística Internacional de Enfermedades y Problemas Relacionados con la Salud (CIE-10), publicada por la Organización Mundial de la Salud, en todos los establecimientos de salud del territorio nacional.

Un componente central de la infoestructura es la historia clínica electrónica (HCE) definida como el registro unificado y personal de datos, valoraciones e informaciones de cualquier índole, sobre la situación y la evolución clínica de un paciente a lo largo del proceso asistencial y refrendado con la firma digital del profesional tratante.

Esta información se encuentra contenida en una base de datos electrónica, registrada mediante programas de computación y cuyo almacenamiento, actualización y uso se efectúa en condiciones estrictas de seguridad, integridad, autenticidad, confidencialidad, exactitud, inteligibilidad, conservación, disponibilidad y acceso.

La Resolución Ministerial 576-2011/MINSA que aprueba la Directiva Administrativa 183 establece las especificaciones para la estandarización del registro en la historia clínica electrónica.

Recientemente mediante la Ley 30024 se estableció la creación del Registro Nacional de Historias Clínicas Electrónicas definida como la infraestructura tecnológica especializada en salud que administra las historias clínicas electrónicas dentro de los términos estrictamente necesarios para garantizar la seguridad y la calidad de la atención a los pacientes. El Registro Nacional de Historias Clínicas Electrónicas (http:// www.minsa.gob.pe/renhice/) contiene una base de datos de filiación de cada persona con la relación de los establecimientos de salud y de los servicios médicos de apoyo que le han brindado atención y de esta manera generaron su historia clínica electrónica. El Ministerio de Salud es el titular de la base de datos contenida en el Registro Nacional de Historias Clínicas Electrónicas y está encargado de su administración. Aún se encuentra pendiente reglamentar esta Ley.

Finalmente, un componente esencial es la interoperabilidad definida como la habilidad de dos o más sistemas o componentes para intercambiar información para posteriormente interpretarla y utilizarla. La interoperabilidad contempla los aspectos técnicos para la interconexión de sistemas de información, a través de la definición de estándares, estructuras de datos y protocolos para la presentación, recojo, intercambio, proceso y transporte de datos según lo establecido en la Resolución Ministerial 537-2011/MINSA.

Para los procesos de intercambio electrónico de datos clínicos y datos administrativos de uso en la atención y cuidados de la salud de las personas se adopta el estándar de interoperabilidad en salud HL7 (Health Level Seven) y se adopta el estándar DICOM (Digital Imaging and Communication in Medicine) para uso en los procesos de intercambio de imágenes medicas, según lo dispuesto en la Resolución Ministerial 576-2011/MINSA.

Este componente también incluye la seguridad, privacidad y confidencialidad de la información. Los sistemas de información deben ser seguros para los pacientes y profesionales de salud. Cuando nos referimos a los pacientes, la seguridad se relaciona con el adecuado resguardo, disponibilidad y acceso de los datos personales. Cuando nos referimos a los médicos, 
la seguridad consiste en que los sistemas implementados no vulneren o comprometan la seguridad jurídica (10)

En el 2011 se publicó la Ley 29733, Ley de Protección de Datos Personales y en el 2013 se aprobó el reglamento de la Ley mediante Decreto Supremo 003-2013-JUS. Además, en el 2013 mediante la Resolución Directoral 019-2013-JUS/ DGPDP se aprobó la Directiva de Seguridad de la Información Administrada por los Bancos de Datos, la cual orienta sobre las condiciones, los requisitos y las medidas técnicas en materia de medidas de seguridad de los bancos de datos.

\section{TELESALUD}

La telesalud es un servicio de salud a distancia que utiliza las TIC para brindar mayor acceso al personal de salud particularmente en áreas rurales, zonas aisladas y con limitada capacidad resolutiva a través de una interconexión con establecimientos de salud que pueden estar localizados en diferentes regiones del Perú (2).

El Perú cuenta con un Plan Nacional de Telesalud (http://www.telesalud.minsa.gob.pe) cuyos elementos son la teleducación que permite la capacitación continua a distancia a través de la discusión virtual de casos clínicos; el telediagnóstico que consisten en la ayuda al diagnóstico del paciente a distancia; la teleconsulta que corresponde al acceso de un teleespecialista en una ubicación remota, y la telegestión que implica reuniones de trabajo a distancia con ejecutivos, especialistas y administrativos (2). Asimismo, la Resolución Ministerial 365-2008/MINSA, considera que la aplicación de la telesalud está involucrado en alguno de los siguientes tres ejes de desarrollo: prestación de servicios de salud o telemedicina, la gestión de servicios de salud, y la información, educación y comunicación a la población y al personal de salud.

En mayo de 2015, la Comisión de Salud y Población del Congreso de la República del Perú aprobó el proyecto de Ley Marco de Telesalud que tiene por objeto establecer los lineamientos generales para la implementación y desarrollo de los servicios de telesalud. El proyecto busca mejorar la eficiencia y la calidad de los servicios de salud e incrementar su cobertura mediante el uso de las TIC.

El Instituto Nacional Materno Perinatal es una de las instituciones del Ministerio de Salud con mayor liderazgo en la telesalud (2), implementando la teleenseñanza desde el año 2008 y conectando a nueve regiones del país mediante una red de telesalud. El Instituto ha implementado un sistema de capacitación virtual en salud materno perinatal y desde el año 2010 se implementó la red nacional de asistencia técnica virtual.

\section{PLANEAMIENTO, EJECUCIÓN, MONITOREO Y EVALUACIÓN}

Todo sistema de información en salud debe contar con un proceso de planeamiento y planificación exhaustiva para su adecuada ejecución. Uno de los componentes esenciales es definir con claridad el presupuesto dedicado a cada uno de los componentes descritos en este marco conceptual, y se debe contemplar que el presupuesto de los servicios TIC incluya partidas para el desarrollo, mantenimiento y evolución de los sistemas de información en salud. Asimismo, es importante tener una definición clara del alcance de los servicios a desarrollar y contratar. Por ejemplo, en cuanto al software, es importante establecer claramente las condiciones de la licencia del producto, la propiedad del código fuente, el entrenamiento de los usuarios, entre otros puntos. Finalmente, el monitoreo y evaluación de cada uno de los componentes es fundamental para una optima gestión de los sistemas de información. Los gestores de proyectos usualmente enfrentan tres grandes desafíos: el cumplimiento de los requerimientos funcionales, el presupuesto asignado y la entrega en las fechas comprometidas ${ }^{(10)}$.

Una buena práctica consiste en implementar un entorno de desarrollo que permitan realizar las diferentes tareas de programación y también implementar un entorno de preproducción y un entorno de producción en el que se aloja el sistema definitivo sobre el que trabajan los usuarios.

Complementariamente a estos componentes que dan soporte a los diferentes procesos de salud, existen dos columnas fundamentales: la primera relacionada a la gobernanza, rectoría, financiamiento, modelo de inversiones y políticas públicas del gobierno; y la segunda relacionada a los recursos humanos capacitados. Por un lado, es fundamental el apoyo al proyecto por la máxima autoridad. Esto permite una mayor capacitad para solucionar situaciones que dificultan el avance apropiado del proyecto ${ }^{(10)}$. La ejecución de un plan en salud electrónica o en sistemas de información debe ser liderada por las máximas autoridades del sector, tanto a nivel ministerial como a nivel local ya que la incorporación de TIC trae consigo cambios profundos en la forma de trabajar y muchas veces lleva a evaluar los 
modelos de atención y los procesos asociados. Asimismo, las organizaciones deben estandarizar sus procesos y deben enfocarse a garantizar costoefectividad de sus procesos.

Además, la implementación y sostenibilidad de un plan de sistemas de información en salud requiere asegurar un financiamiento importante durante un período prolongado.

Por otro lado, es clave fortalecer destrezas y conocimientos para utilizar adecuadamente los sistemas de información en salud. Las dificultades para una implementación efectiva de un sistema pueden dar lugar a resistencias para la incorporación del nuevo sistema. Es básico que las personas que operen y utilicen el sistema se capaciten apropiadamente con anticipación a su implementación. En lo posible, los usuarios finales deben estar involucrados desde las etapas iniciales del desarrollo de un sistema de información, considerando las metodologías de usabilidad y diseño centrado en los usuarios.

Finalmente, se necesita generar una política de incentivos adecuados para que la implementación de los sistemas de información en salud se ejecute según lo planificado, y es necesario que se implemente apropiadamente una estrategia comunicacional y de gestión del cambio, considerando que los proyectos de informatización en el sector salud suelen ser proyectos complejos.

\section{POLÍTICAS NACIONALES RELACIONADAS A LA SALUD ELECTRÓNICA}

El marco conceptual se relaciona con otras políticas nacionales como el "Proceso de Modernización de la Gestión Pública al 2021" (11). Para esta política el protagonista es el ciudadano y su finalidad es que estos reciban bienes y servicios de calidad al menor costo posible.

Un estado moderno implica que sea: orientado al ciudadano, que asigna sus recursos y se organiza en función de las necesidades de la ciudadanía; eficiente, que utiliza sus recursos de manera racional asegurando que los ciudadanos accedan a bienes y servicios de calidad, en cantidades óptimas y al menor costo posible; unitario y descentralizado, que se adapta a las diferentes necesidades de cada territorio trabajando de la mano con los gobiernos descentralizado; es inclusivo, es decir, que busca que todos gocen de las mismas oportunidades y accedan a bienes y servicios de calidad, y es abierto, transparente, que fomenta la participación ciudadana, promueve la ética pública y rinde cuentas de su desempeño.

El Plan Nacional de Gobierno Electrónico 2013-2017, preparado por la Oficina Nacional de Gobierno Electrónico e Informática (ONGEI), incluye 28 proyectos de gobierno electrónico de los cuales seis están relacionados al sector salud y se ha encomendado al Ministerio de Salud, como ente rector del sistema, el desarrollo e implementación de los mismos para el período 2013-2017 (12). Los proyectos que se incluyen son el sistema de Registro de Nacidos Vivos en Línea que permite registrar en la misma sala de partes de los establecimientos de salud públicos y privados ${ }^{(13)}$; telesalud en zonas de frontera y aisladas cuyo objetivo es fortalecer las capacidades del personal de salud de los establecimientos de salud ubicados en zonas de la frontera nacional a través de servicios de telemedicina con conectividad y plataforma de videoconferencia, además de dotarlos con sistemas y aplicativos propios del sector con la finalidad que se brinden servicios de salud de manera eficiente a dicha población; telesalud para establecimientos de salud priorizados; hacia la historia clínica electrónica; sistema de referencias y contrarreferencias en el sector salud con la finalidad que agilizar y reducir el porcentaje de referencias no justificadas a través de conectividad, plataforma integrada de videoconferencia, sistema de archivado, transmisión de imágenes y dispositivos.

La Agenda Digital Peruana $2.0{ }^{(14)}$, desarrollada por la Comisión Multisectorial para el Seguimiento y Evaluación del Plan de Desarrollo de la Sociedad de la Información en el Perú (Decreto Supremo 066-2011PCM) tiene como meta principal permitir que la sociedad peruana acceda a los beneficios que brinda el desarrollo de las tecnologías de la información y comunicación en todos sus aspectos. De los ocho objetivos de este documento, el tercero hace mención a Garantizar mejores oportunidades de uso y apropiación de las TIC que aseguren la inclusión social, el acceso a servicios sociales que permita el ejercicio pleno de la ciudadanía y el desarrollo humano en pleno cumplimiento de las metas del milenio. Este objetivo tiene como primera estrategia fortalecer la salud de la población en sus componentes de persona, familia y comunidad mediante el uso intensivo de las TIC. Es necesario impulsar una gestión de salud que lleve a una mayor conectividad de los hospitales y centros de salud públicos del país. Para ello, además, se debe promover el desarrollo y correcto uso de sistemas de información para mejorar la gestión hospitalaria, como por ejemplo, sistemas de citas médicas en línea (http://www. minsa.gob.pe/citas/), interconsultas electrónicas, emisión de recetas médicas electrónicas, organización de la gestión hospitalaria regular y de emergencias, entre otros, 
centrados en el usuario de salud. Todo ello implicará, entre otros esfuerzos, implementar enfoques de interoperabilidad a las plataformas públicas de salud.

\section{LA REFORMA EN SALUD Y SU \\ RELACIÓN CON EL MARCO \\ CONCEPTUAL PARA FORTALECER \\ LOS SISTEMAS DE INFORMACIÓN EN SALUD EN EL PERÚ}

Varios componentes del marco conceptual han sido incluidos en los lineamientos de reforma del sector salud ${ }^{(15)}$. Así, el lineamiento 2 , relacionado a fortalecer la atención primaria en salud, menciona la ejecución de un sistema de información hospitalaria y de intercambio de datos entre los establecimientos de salud del Ministerio de Salud, los gobiernos regionales, EsSalud, las Fuerzas Armadas y la Policía Nacional ${ }^{(15) .}$

Por otro lado, el lineamiento 3, relacionado con mejorar la eficiencia, calidad y acceso a servicios hospitalarios especializados, considera que el personal de salud pueda tener acceso a actividades de capacitación por medios tecnológicos modernos y a la telemedicina. Además, establece que se deberá establecer una red de servicios de telemedicina, con énfasis inicial en la atención materno-neonatal y el cáncer, para conectar especialistas con médicos tratantes de los establecimientos de salud en las regiones del Perú ${ }^{(15)}$.

Además, el lineamiento 4 relacionado con reformar la política de gestión de recursos humanos desarrolla las posibilidades que ofrecen las nuevas tecnologías, como la telemedicina, y los sistemas de información, con una gran capacidad de conectividad, robustos, flexibles y apoyados en estándares técnicos internacionales de referencia. La propuesta de mandato de política 4.10 establece la construcción de un sistema de información integrado orientado al gobierno y conducción del sector salud. Se reconoce la necesidad de aplicar un sistema de información en salud integrado para brindar información en salud en la base de identificadores estándares de datos en salud que sea confiable, consistente y oportuno, orientado a convertirse en una herramienta eficaz para el gobierno y la conducción sectorial de las políticas públicas en salud. Se plantean cuatro arreglos clave para la implementación (15):

1. El Ministerio de Salud deberá diseñar y ejecutar un plan de desarrollo del sistema nacional de información en salud en dos fases. La primera, para dar cumplimiento de los identificadores estándares de datos en salud, especificados en el Decreto Supremo 024-2005-SA, y progresivamente con el conjunto de normas técnicas y estándares de referencia nacionales para los sistemas de información en salud.

2. El Ministerio de Salud deberá desarrollar e implementar un repositorio nacional de información en salud, de forma concertada con los actores del sector salud.

3. EL Ministerio de Salud deberá elaborar un plan nacional para la implementación de la telesalud y la telemedicina, y del certificado de nacido vivo en línea, de forma concertada con los actores del sector salud.

4. El Ministerio de Salud promoverá el desarrollo e implementación de una plataforma transaccional y la historia clínica electrónica sectorial que permita implementar el Registro Nacional de Historias Clínicas Electrónicas ${ }^{(15)}$.

\section{CONCLUSIÓN}

Las TIC pueden aportar a una mejor gestión de la salud mediante desarrollos, como el referido a la telesalud, considerando nuestro territorio tan vasto como accidentado, y donde los servicios de salud requieren de opiniones especializadas que, en muchos casos, se encuentran a cientos de kilómetros, y por tanto, las TIC pueden aportar a la mejora en la cobertura y la calidad, beneficiando, sobre todo, a los sectores más vulnerables.

En conclusión, los componentes descritos para fortalecer los sistemas de información en salud en el Perú cumplen un rol clave y se espera lograr un sistema de información integrado e interoperable que permita que la información en salud sea lo más completa, eficiente, de calidad y esté disponible oportunamente, para lograr mejorar la calidad de vida de las personas y permitir una modernización significativa de la salud pública en el marco de la reforma en salud en el Perú.

Contribución de los autores: WHC y EE participaron en la redacción del manuscrito y aprobaron la versión final a publicar.

Fuentes de financiamiento: autofinanciado.

Conflictos de interés: los autores declaran no tener conflictos de interés en la publicación de este artículo. 


\section{REFERENCIAS BIBLIOGRÁFICAS}

1. Wagenaar BH, Sherr K, Fernandes Q, Wagenaar AC. Using routine health information systems for well-designed health evaluations in low- and middleincome countries. Health Policy Plan. 2015 Apr 16. pii: czv029.

2. Curioso WH. eSalud en Perú: implementación de políticas para el fortalecimiento de sistemas de información en salud. Rev Panam Salud Publica. 2014;35(5-6):437-41.

3. Perú, Ministerio de Salud. Resolución Ministerial 297-2012/ MINSA: Establecimiento del Marco Conceptual para el Fortalecimiento en Sistemas de Información y Tecnologías de Información y de Comunicación en el Ministerio de Salud. El Peruano. 17 de abril del 2012. 464274 (col. 1).

4. World Health Organization, Health Metrics Network. Framework and standards for country health information systems. 2da ed. Geneva: WHO; 2008.

5. International Organization for Standardization. Techincal Report 14639-1: Part 1: Capacity-based eHealth architecture roadmap: Overview of national eHealth initiatives [Internet]. Geneva: ISO; 2012 [citado el 2 de marzo de 2015]. Disponible en: https://www.iso.org/ obp/ui/\#iso:std:iso:tr:14639:-1:ed$1: \mathrm{v} 1: \mathrm{en}$
6. Rojas D, Martinez R, Elicegui I. Infraestructura y requisitos básicos de los sistemas de salud electrónica. En: Naciones Unidas; Carnicero J, Fernández A. Manual de salud electrónica para directivos de servicios y sistemas de salud. Santiago de Chile: Naciones Unidas; 2012. p. 365-83.

7. Díaz Ataucuri D, Guadalupe Sifuentes I, Chamorro R, Tucto L, Curioso WH. Despliegue de infraestructura de fibra óptica para formar la Red Nacional de Investigación y Educación en el Perú. Cuarta Conferencia de Directores de Tecnologías de Información y Comunicación de Instituciones de Educación Superior, TICAL 2014, Cancún, México..26-28 de mayo de 2014.

8. Tsiknakis M, Katehakis DG, Orphanoudakis SC. An open, component-based information infrastructure for integrated health information networks. Int J Med Inform. 2002 Dec;68(1-3):3-26.

9. Alvarez RC. The promise of e-Health - a Canadian perspective. eHealth Int. 2002 Sep 17;1(1):4.

10. Orellana R. Gestión de proyectos de salud electrónica. En: Naciones Unidas; Carnicero J, Fernández A. Manual de salud electrónica para directivos de servicios y sistemas de salud. Santiago de Chile: Naciones Unidas; 2012. p. 345-63.

11. Perú, Secretaría de Gestión Pública - Presidencia del Consejo de Minis- tros. Política Nacional de Modernización de la Gestión Pública al 2021. Lima: SGP-PCM; 2013.

12. Perú, Oficina Nacional de Gobierno Electrónico e Informática. Política Nacional de Gobierno Electrónico 2013-2017. Lima: ONGEI; 2013.

13. Curioso WH, Pardo K, Loayza M. Transformando el sistema de información de nacimientos en el Perú. Rev Peru Med Exp Salud Publica. 2013;30(2):303-7.

14. Comisión Multisectorial para el Seguimiento y Evaluación del Plan de Desarrollo de la Sociedad de la Información. Decreto Supremo 0662011-PCM: Plan de Desarrollo de la Sociedad de la Información en el Perú - La Agenda Digital Peruana 2.0. Lima, Perú. 26 de julio 2011.

15. Perú, Ministerio de Salud. Lineamientos y medidas de reforma del sector salud. Lima: MINSA; Julio 2013.

Correspondencia: Walter H. Curioso Dirección: Department of Biomedical Informatics and Medical Education, School of Medicine, University of Washington. Seattle, Washington, EE. UU.

Correo electrónico: wcurioso@uw.edu; waltercurioso@gmail.com

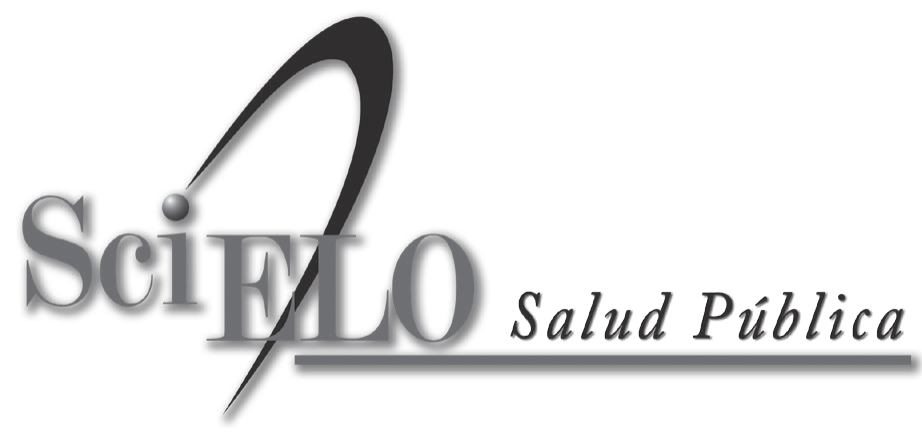

